\title{
Reducing Disparities in the Quality of Palliative Care for Older African Americans through Improved Advance Care Planning: Study Design and Protocol
}

\author{
Deborah B. Ejem, $\mathrm{PhD},{ }^{1}$ Nadine Barrett, $\mathrm{PhD},{ }^{2}$ Ramona L. Rhodes, MD, MPH, ${ }^{3}$ Maren Olsen, $\mathrm{PhD},{ }^{4,5}$ \\ Marie Bakitas, DNSc, CRNP, ${ }^{6,7}$ Raegan Durant, MD, MPH, ${ }^{1}$ Ronit Elk, PhD, ${ }^{7}$ Karen Steinhauser, PhD, \\ Tammie Quest, MD, ${ }^{8}$ Rowena J. Dolor, MD, MHS, ${ }^{2}$ and Kimberly Johnson, MD ${ }^{9}$
}

\begin{abstract}
Advance care planning (ACP) improves end-of-life care for patients and their caregivers. However, only one-third of adults have participated in ACP and rates are substantially lower among African Americans than among whites. Importantly, ACP improves many domains of care where there are racial disparities in outcomes, including receipt of goal-concordant care, hospice use, and provider communication. Yet, few studies have examined the effectiveness of ACP interventions among African Americans. The objectives of reducing disparities in the quality of palliative care for older African Americans through improved advance care planning (EQUAL ACP) are as follows: to compare the effectiveness of two interventions in (1) increasing ACP among African Americans and whites and (2) reducing racial disparities in both ACP and end-of-life care; and to examine whether racial concordance of the interventionist and patient is associated with ACP. EQUAL ACP is a longitudinal, multisite, cluster randomized trial and a qualitative study describing the ACP experience of participants. The study will include 800 adults $\geq 65$ years of age (half African American and half white) from 10 primary care clinics in the South. Eligible patients have a serious illness (advanced cancer, heart failure, lung disease, etc.), disability in activities of daily living, or recent hospitalization. Patients are followed for one year and participate in either a patient-guided, self-management ACP approach, including a Five Wishes form or structured ACP with Respecting Choices First Steps. The primary outcome is formal or informal ACP-completion of advance directives, documented discussions with clinicians, and other written or verbal communication with surrogate decision makers about care preferences. Secondary outcomes assessed through after-death interviews with surrogates of patients who die during the study include receipt of goal-concordant care, health services use in the last year of life, and satisfaction with care. EQUAL ACP is the first large study to assess which strategies are most effective at both increasing rates of ACP and promoting equitable palliative care outcomes for seriously ill African Americans.
\end{abstract}

Keywords: advance care planning; African Americans; end of life; five wishes; racial disparities; respecting choices

\section{Introduction}

A LTHOUGH ALL SERIOUSLY ILL PATIENTS are at risk for poor quality care, the risk is even greater among African Americans. ${ }^{1-3}$ In the last months of life, compared to whites,
African Americans more commonly experience high-cost, low-quality care characterized by untreated pain, avoidable hospitalizations, poor communication with providers, receipt of care inconsistent with their preferences, and lower rates of hospice enrollment. ${ }^{1-3}$ These disparities are likely to worsen

\footnotetext{
${ }^{1}$ Division of Preventive Medicine, School of Medicine, University of Alabama at Birmingham, Birmingham, Alabama.

${ }^{2}$ Deparment of Community and Family Medicine, Duke University School of Medicine, Durham, North Carolina.

${ }^{3}$ Department of Internal Medicine, University of Texas Southwestern Medical Center, Dallas, Texas.

${ }^{4}$ Biostatistics and Bioinformatics, Duke University School of Medicine, Durham, North Carolina.

${ }^{5}$ Center for Health Services Research, Durham VA Medical Center, Durham, North Carolina.

${ }^{6}$ School of Nursing, University of Alabama at Birmingham, Birmingham, Alabama.

${ }^{7}$ Division of Geriatrics, Gerontology, and Palliative Care, School of Medicine, University of Alabama at Birmingham, Birmingham, Alabama.

${ }^{8}$ Department of Emergency Medicine, Emory University School of Medicine, Atlanta, Georgia.

${ }^{9}$ Division of Geriatrics, Department of Medicine, Center for the Study of Aging and Human Development, Center for Palliative Care Duke University School of Medicine and Geriatrics Research Education and Clinical Center, Durham VA Medical Center, Durham, North Carolina. Accepted June 13, 2019.
} 
with the growing population of older adults. Between 2015 and 2030, the number of adults who are 65 years of age and older is expected to increase from 50 million to over 70 million. ${ }^{4,5}$ While the proportion of non-Hispanic whites will increase by 59\%, the proportion of older African Americans (largest minority group of older adults) will increase by $115 \% .{ }^{1,5}$ Because older African Americans have worse selfreported health, more disability, and higher rates of serious life-limiting illness, targeted approaches are needed to improve the quality of care for this vulnerable population. ${ }^{6-8}$

Advance care planning (ACP) improves many domains of care where there are racial disparities in outcomes, including receipt of goal-concordant care, provider communication, family satisfaction, preventable hospitalization, and hospice use. $^{7,9}$ Among caregivers of patients who die, ACP is also associated with less depression and anxiety. Despite the benefits of ACP and the beliefs among the vast majority of Americans, including African Americans, that ACP is important, as few as $30 \%$ of adults have participated in ACP. ${ }^{7,9}$ Rates of ACP are substantially lower among African Americans than whites across age groups. ${ }^{1,3,7,10}$ Currently, we do not know the extent to which these differences reflect fixed cultural or individual values or modifiable community-based, provider, and health system factors.

Although a large body of research has examined factors that may explain lower rates of ACP among African Americans, ${ }^{7,10-12}$ few studies have examined the effectiveness of interventions to promote ACP for this group. Current research is limited by small samples that focus on a single disease population and include short-term follow-up. To date, no large studies have compared the effectiveness of two or more widely used or evidence-based interventions to facilitate equitable ACP and improve care for seriously ill African Americans and their caregivers. ${ }^{9,13,14}$

$\mathrm{ACP}$, among African Americans, is influenced by a complex interaction of historical, personal, interpersonal, and system factors, including sociodemographics, mistrust in the health care system, access to care, religion and spirituality, family, community, and clinician interactions. ${ }^{10,15}$ Based on these factors, the current opportunities to improve ACP for African Americans using existing interventions include the following: (1) adopting a broader view of ACP with less emphasis on legal directives; (2) ensuring equal access to ACP interventions; (3) use of interventions that increase ACP knowledge and consider health literacy; (4) creating trusting relationships with peers; (5) and addressing clinician barriers to ACP through the use of processes that do not rely on them to initiate ACP. With this framework, we developed the study, Reducing Disparities in the Quality of Palliative Care for Older African Americans through Improved Advance Care Planning (EQUAL ACP). This article describes the study protocol for EQUAL ACP, a comparative effectiveness trial of two ACP interventions among seriously ill, older African Americans and whites.

\section{Materials and Methods}

\section{Study overview}

The overall goal of EQUAL ACP is to compare the effectiveness of two different ACP interventions in increasing $\mathrm{ACP}$ and promoting equitable palliative care outcomes among seriously ill, older African Americans and whites. EQUAL ACP includes a longitudinal, multisite, matched pair (patient and surrogate decision maker), cluster randomized trial and a qualitative study (semistructured interviews and analysis of ACP conversations) to describe the ACP experience of participants. The study will include 800 older adults residing in 5 states.

This study protocol was reviewed and approved by Institutional Review Boards (IRBs) across study sites, including Duke University Health System IRB (Pro00091633), which serves as central IRB for University of Alabama at Birmingham and two federally qualified health centers in South Carolina; Emory University IRB (IRB00103144); and University of Texas Southwestern (STU 052018-047). Study participants receive $\$ 50$ for each data collection survey (four time points - total of \$200), qualitative interview (random sample), or after-death interview (surrogates of patients who die during study).

\section{Objectives and hypotheses}

The primary aims of EQUAL ACP are to compare the effectiveness of a structured ACP approach to a patientguided, self-management approach in the following: (1) facilitating ACP among older adults within each racial subgroup (African American and whites) and (2) reducing disparities in ACP between racial subgroups. Our secondary aims are to (1) determine if racial concordance of patient and lay ACP facilitator is associated with the effectiveness of the intervention in promoting ACP; and (2) among decedents, compare the effectiveness of the two ACP interventions in reducing racial disparities in receipt of care consistent with patient preferences, caregiver distress, satisfaction with care, and health care utilization in the last six months of life. The aim of the qualitative component is to describe racial differences in the experience of ACP, including beliefs, values, and goals, which may inform approaches to ACP.

EQUAL ACP will test the following hypotheses: (1) compared to a patient-guided, self-management approach, a structured ACP approach will result in the following: (a) higher rates of ACP among study participants within each racial subgroup and (b) smaller differences in rates of ACP between racial subgroups; and among decedents, smaller racial differences between subgroups in patient and caregiver outcomes. (2) Study participants in both racial subgroups who receive either ACP intervention from a lay ACP facilitator of the same race (vs. of a different race) will have higher rates of ACP.

\section{Setting}

We will enroll patients receiving care in 10 clinics in 5 states (2 clinics in each state), including Alabama, Georgia, North Carolina, Texas, and South Carolina. These sites are in the Deep South, an area where more than one-third of African Americans reside and where disparities in health outcomes are most pronounced. ${ }^{5,16}$ With the exception of the two federally funded health centers in South Carolina, all clinics are affiliated with an academic medical center. All the clinics are primary care practices and one is a geriatric medicine practice.

\section{Population}

Table 1 includes eligibility criteria of participants. The criteria capture older adults with serious illness defined as those at high risk of mortality, functional decline, or 
Table 1. Reducing Disparities in the Quality of Palliative Care for Older African Americans through Improved Advance Care Planning Eligibility Criteria

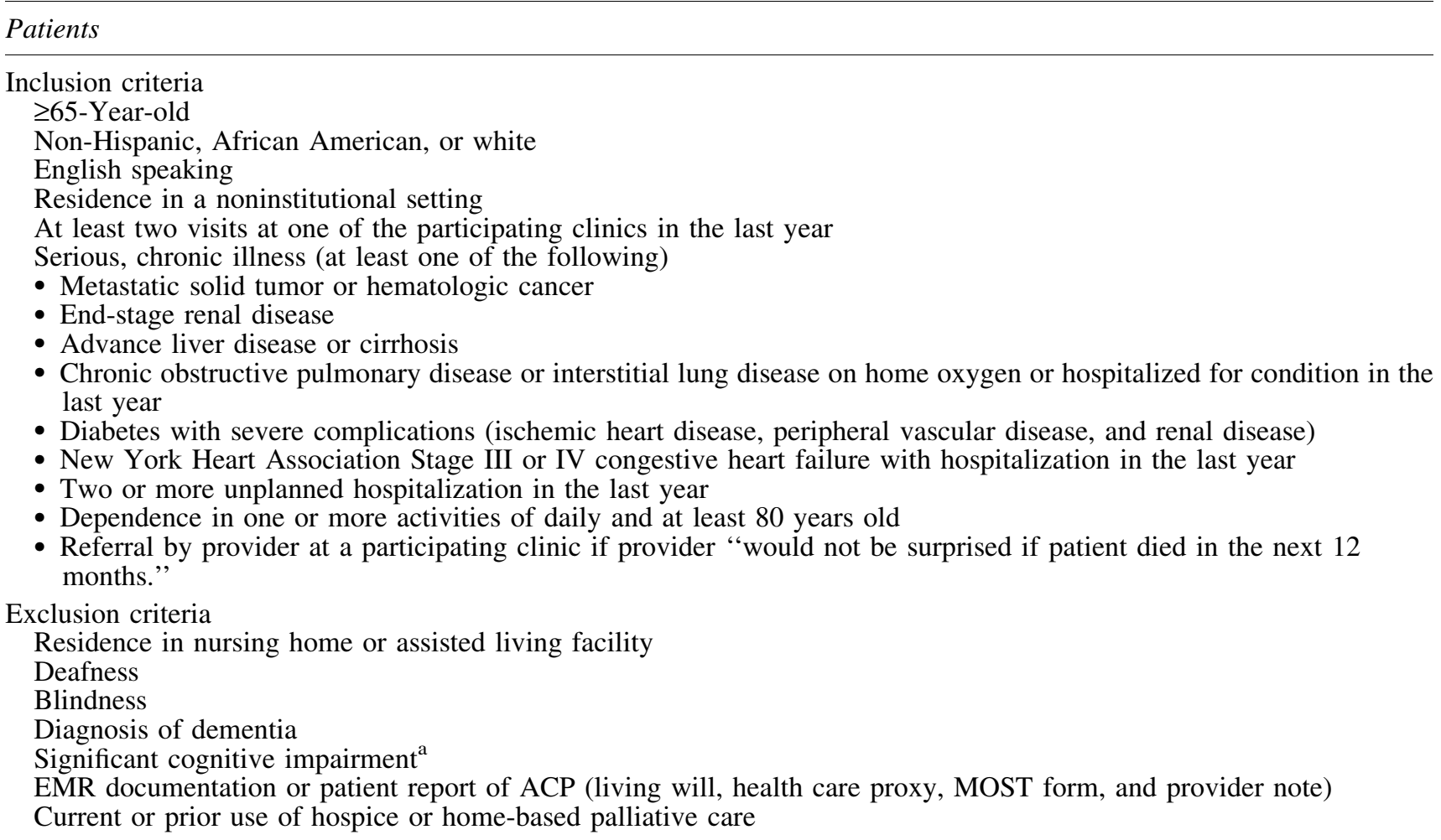

Caregiver/surrogate decision maker

Inclusion criteria

$\geq 18$ Years old

Named by enrolled patient who provides contact information and permission to contact

Exclusion criteria

Significant cognitive impairment ${ }^{\mathrm{a}}$

Not be available to complete scheduled data collection by phone over next year

\footnotetext{
${ }^{a}$ Assessed by telephone screen as score greater than two on six-item cognitive screener. ${ }^{20}$
}

ACP, advance care planning; EMR, electronic medical record.

hospitalization. ${ }^{17}$ Patients are eligible if they are English speaking, community dwelling, $\geq 65$ years of age, nonHispanic, and white or African American and have a serious illness (advanced cancer, renal, liver, lung, or heart disease), and two or more unplanned hospitalizations in the last year; and $\geq 80$ years of age along with dependence in one or more activities of daily living. Providers may refer patients to the study regardless of diagnosis if they "would not be surprised if patient died in the next 12 months.",18,19 Although the surprise questions are a poor screening tool for mortality in a primary care population, it has been significantly associated with one-year mortality over and above age, gender, and comorbidities when used by primary care physicians to identify patients at high risk of death. ${ }^{19}$ We added the surprise question to our eligibility criteria to allow providers to refer patients to the study, who may not be identified by our electronic medical record (EMR) screening, but whom they believe are at high risk of death. Patients are excluded if they have an ACP (documented in EMR or reported by patient), reside in an institution, or would not be able to take part in the study protocol because of significant sensory or cognitive impairment. ${ }^{20}$
Enrolled patients are asked to identify one person who would help to make medical decisions for them if they become unable to make their own decisions. This may or may not be a person who also serves as a caregiver, assisting them with day-to-day care. Caregivers or surrogate decision makers who are named by the patient undergo telephone screening and are eligible to participate if they are $\geq 18$ years of age and cognitively able to participate. Patients who do not identify a surrogate or caregiver or whose surrogates decline participation will still be allowed to participate.

\section{Recruitment}

Figure 1 includes the EQUAL ACP study flow. Potentially eligible patients with an upcoming appointment (three to four weeks out) at a participating clinic are identified by administrative data pull. Study personnel at each site screen EMRs to assess eligibility, and potentially eligible patients are mailed a letter from their primary care provider introducing the study. Patients who do not opt out of further contact by returning a post card or calling study office undergo 


\section{Administrative Data Pull and Chart Review}

- Non Hispanic African Americans and Whites

- $\quad \geq$ age 65

- Visit at participating practices in next 3 to 4 weeks

- Chart Review to assess eligibility (Table 1 for inclusion/exclusion criteria)

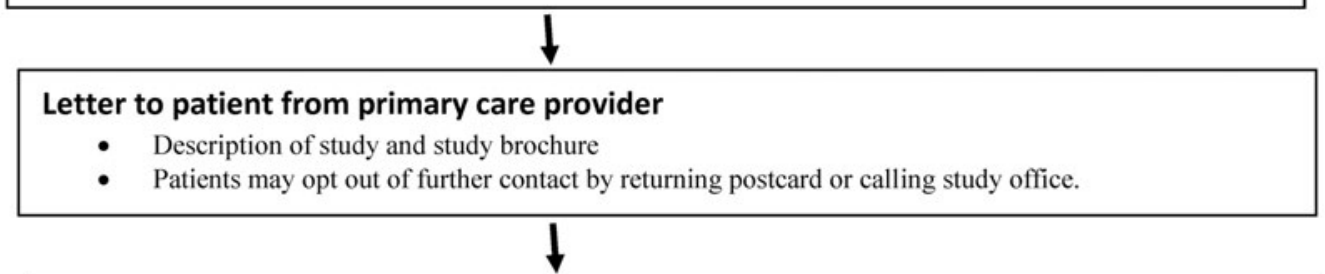

\section{Follow-up Telephone Screen}

- Verify ethnicity, race, residence, absence of advance directive, absence of prior hospice or homebased palliative care use, assess cognition

- Assess ADL disability in patients $\geq 80$ without serious illness

Consent patient, complete baseline assessment, identify surrogate decision maker

- Patient consent can be completed at upcoming clinic visit or by phone

- Patient asked to identify a person who is likely to assist with medical-decision making and provide contact information

- $\quad$ Surrogate contacted, screened, consented, and baseline completed

Block randomization within clinics to ACP interventionist based on patient race

- Patient randomized to interventionist of same race or different race

- Patient receives intervention materials and contact card of ACP interventionists

\section{Patient-driven, Self-management ACP using Five Wishes}

- Phone contact by interventionist to review materials and answer questions two weeks after materials received.

- Follow-up phone call by interventionist 4 weeks after initial call.

- Optional additional phone call if requested
Structured ACP Approach using Respecting Choices First Steps

- Phone contact by interventionist to schedule Respecting Choices First Step ACP facilitation session two weeks after materials received.

- ACP facilitation using Respecting Choices First Step (inperson if possible; if not, by phone)

- Follow-up phone call by interventionists two weeks after the ACP session.

- Optional additional phone call if requested

\section{After Death Interviews}

- Completed by enrolled surrogate or other person knowledgeable about last month of life (3 to 6 months after death of patient)

- Chart review for healthcare utilization (hospitalization, ED visits, use of hospice and palliative care) in the last year of life

FIG. 1. EQUAL ACP study flow. EQUAL ACP, reducing disparities in the quality of palliative care for older African Americans through improved advance care planning. 
telephone screening. Patients who are eligible and interested in participating complete informed consent. Once enrolled, patients provide name and contact information of a surrogate decision maker who is contacted by phone to complete eligibility screening and informed consent if he or she is interested in participating.

\section{Intervention}

Based on input of our stakeholders, we identified two ACP approaches for comparison: (1) a structured ACP approach using Respecting Choices First Steps and (2) a patient-driven, self-management approach, including a Five Wishes form. Both approaches are delivered by lay ACP facilitators. In addition to the materials and intervention described below, all participants receive state advance directive forms and a contact card with telephone number and picture of their assigned lay ACP facilitator.

Respecting Choices is an ACP program that has been implemented worldwide. Studies of Respecting Choices have shown an increase in the prevalence of advance directives, greater patient-surrogate congruence for treatment preferences, receipt of goal-concordant care, and decrease in health care utilization. ${ }^{21-24}$ A recent systematic review of Respecting Choices and related models noted limitations in research methods and inconsistency in findings related to ACP outcomes across published studies. ${ }^{25}$ In addition, evidence on its use and outcomes in minority populations is limited. ${ }^{21,22}$ The Respecting Choices model includes three stages of ACP, First Steps, Next Steps, and Last Steps. First Steps is intended for those who have not planned. Next Steps involves ACP for those with advanced illness, and Last Steps involves expressing preferences as medical orders through the POLST paradigm. Although we are enrolling older adults with serious illness, we believe that First Steps is appropriate because (1) our study will include older adults who have not previously participated in ACP. First Steps is intended for those who have not planned. (2) A focus on specific treatments (Last Steps), rather than ACP as a process for sharing wishes for care in any way you choose, may not be consistent with values of some African Americans. ${ }^{7}$ (3) ACP is not a one-time event. The goal of the intervention is to start to engage patients in ACP. Participants are encouraged to discuss their wishes with their providers who can focus more specifically on their disease, prognosis, and potential treatment options. Our intervention is one way to start to engage patients in ACP. (4) To ensure wide dissemination and rapid implementation of our findings, we are using lay facilitators who may have limited knowledge of specific disease processes.

Participants receive Respecting Choices materials that include general information about ACP and choosing a health care agent. Within two weeks, the patient is contacted by a lay ACP facilitator who sets up a time to meet with patient and surrogate decision maker for a 60-90-minute ACP session. The conversation focuses on identifying cultural, spiritual, and personal beliefs that influence treatment preferences, identifying a health care agent, and exploring goals for medical care. These meetings occur in person if possible (by phone if not). The lay ACP facilitator follows up with a phone call two weeks after the meeting to answer questions. Patients may request one additional follow-up call (Fig. 1).

Patients at clinics randomized to the patient-driven, selfmanagement ACP approach receive the Five Wishes advance directive form and the Five Wishes Conversation Guide for Individuals and Families. Although Five Wishes has not been systematically studied, it is the most widely used advance

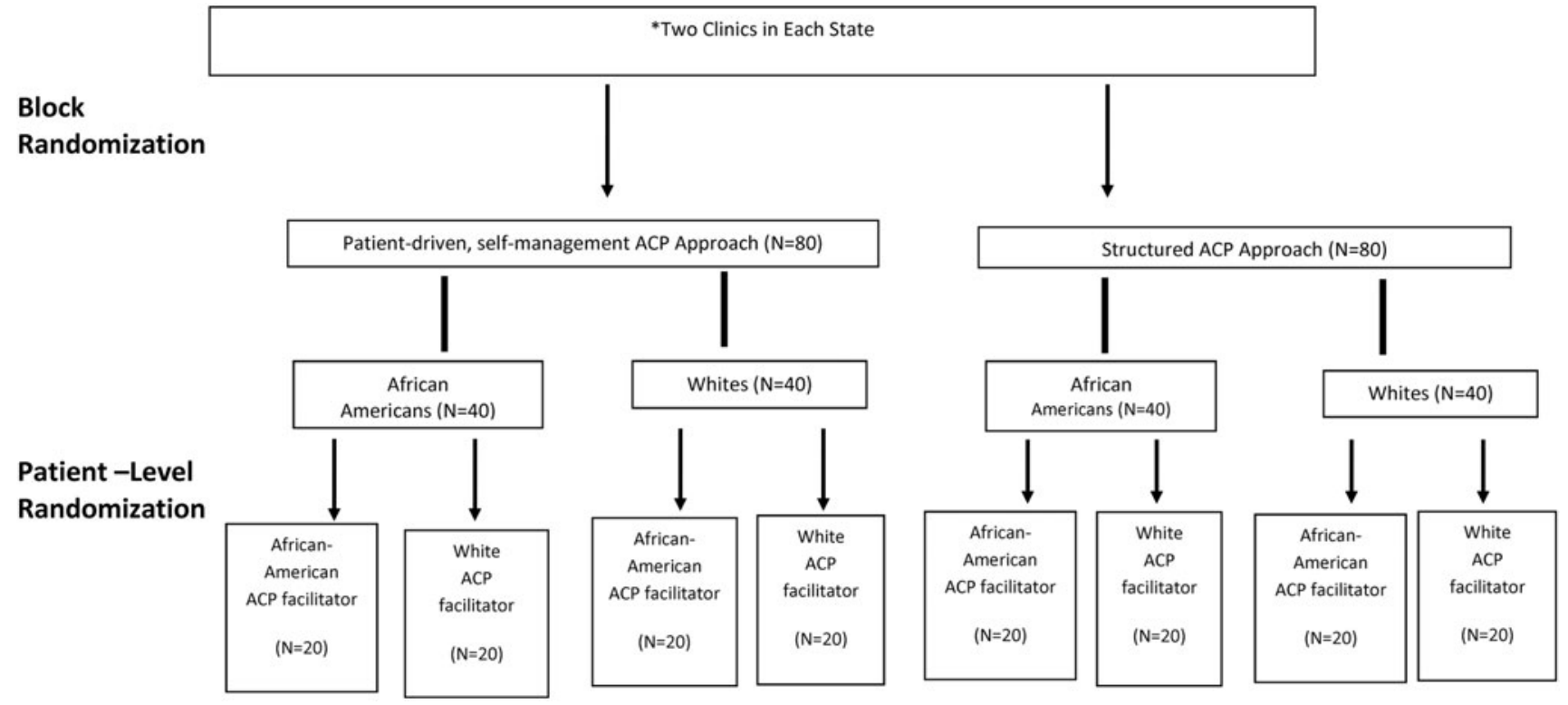

FIG. 2. EQUAL ACP randomization scheme. There are a total of 10 clinics, 2 in each of 5 states. This figure shows randomization recruitment and for two clinics in one state. The two clinics in each state were randomly assigned to either the patient-driven self-management or the structured ACP approach through a blocked randomization scheme. We will enroll 40 African American and 40 white patients from each clinic for a total of 800 patients, half in each racial group. Within each clinic and racial group, patients are randomized in a 1:1 ratio to have the intervention delivered by either a racially discordant or concordant lay ACP facilitator. Thus, half of the patients in each racial group will receive the intervention from an ACP facilitator of their race and half from an ACP facilitator of a different race. ACP, advance care planning. 
directive in America with over 25 million copies distributed by 40,000 organizations. ${ }^{26}$ Five Wishes is both a decision aid and an advance directive written in nontechnical language. The document allows patients to share their "wishes" for the following: the person they would like to serve as surrogate decision maker; the kind of medical care that they would like to receive; how comfortable they want to be; how they want people to treat them; and what they want their loved ones to know. Five Wishes is a legal directive in three of the five states (North Carolina, South Carolina, and Alabama) from which we will recruit patients, and can be used along with state directive forms in the other states. ${ }^{26}$ Two weeks after participants receive the Five Wishes materials, a lay ACP facilitator contacts them by phone to review the materials and answer questions using a scripted protocol that we developed. The lay facilitator follows up four weeks later to answer questions and is available for an optional third call if requested (Fig. 1).

\section{Randomization}

EQUAL ACP is a cluster randomized trial, with randomization occurring at both the clinic (i.e., the cluster) and the patient level (Fig. 2). The two clinics in each state were randomly assigned to either the patient-driven self-management or the structured ACP approach through a blocked randomization scheme, with site as the block. We will enroll 40 African American and 40 white patients from each of the 10 clinics, for a total of 800 patients, half in each racial group. Within each clinic and racial group, patients are randomized in a 1:1 ratio to have the intervention delivered by either a racially discordant or concordant lay ACP facilitator. Thus, half of the patients in each racial group (200 in total) will receive the intervention from an ACP facilitator of their race and half from an ACP facilitator of a different race. Clinic-level and patient-level randomization schemes were developed in PROCPLAN (SAS v 9.4).

\section{Outcome measures}

Study measures are detailed in Table 2 . The primary outcome for EQUAL ACP is formal or informal ACP one year after the intervention. Based on discussions with stakeholders (discussed in more detail in Patient and Key Stakeholder Engagement section), we adopted a broad definition of ACP. We defined ACP as any of the following: (1) completion of a formal document (HCPOA, living will, Five Wishes, POLST, or other legal directive) or an informal document (i.e., letter) naming a decision maker or describing preferences; (2) discussion with clinician documented in chart; and (3) patient report that he/she asked someone to make decisions for him/ her or discussed values, goals, or preferences for future medical care with family, friends, or other surrogate decisionmakers. We believe that this broad definition is the most patient-centered approach because it accounts for the fact that patients vary in how and with whom they choose to express their preferences for care. This is especially relevant for African Americans who are more likely to discuss their preferences with others than to complete advance directives. ${ }^{7}$

Our secondary outcomes are proximal, intermediate, and distal measures of the effectiveness of the ACP process. These include readiness to engage in ACP, beliefs about ACP, congruence between patients and surrogate decision makers regarding patients' preferences, and surrogates' comfort with end-of-life decision-making for patient. For patients who die during the study period, we will collect information on health care utilization (acute care use and palliative care use) during the last six months of life, and surrogates' satisfaction with end-of-life care and surrogate mental health outcomes.

In addition to our outcome measures, we included a number of measures to assess patient and caregiver factors that may both influence ACP and differ by racial groups. These include demographics, function, health literacy, trust in providers and the health care system, religiosity/spirituality, and perceived barriers to ACP.

\section{Data collection}

Our outcome measures will be assessed at baseline, three months, six months, and one year (Table 2). For patients who die, we will interview an enrolled surrogate or next-of-kin three to six months after patient's death. We developed a centralized tool for capturing data across study sites electronically using a custom participant tracking application and REDCap. ${ }^{27}$ Trained clinical trial coordinators at each site collect and enter data into REDCap in real time. The tracking application and REDCap projects are housed on a secure database server at Duke with access limited to key personnel. Throughout enrollment and data collection, we will monitor data quality, accuracy, and timeliness.

\section{Qualitative data collection}

We will describe racial differences in the experience of the $\mathrm{ACP}$ process using qualitative analysis of a random sample of recordings of ACP conversations between patients and interventionists and interviews with participants from clinics in both arms of the study. The interviews will be completed after the six-month data collection using a semistructured interview guide to facilitate a thick description of the ACP experience in participants' own words, including barriers and facilitators to ACP; role of trust, spirituality, and family; and an evaluation of the interventions to identify the most and least helpful elements as well as additional components, which may be important to participants. We expect our final sample will include analyses of $~ 50$ African Americans and 50 whites (patients and caregivers) for each data collection method (ACP conversations and interviews) with purposive sampling based on sociodemographic factors, study site, and racial concordance between patient and ACP facilitator. All recordings will be transcribed and entered into Atlas.ti for data management. ${ }^{28}$ We will analyze the data using techniques of directed content analysis, organizing the data, and identifying common themes in response to the a priori questions contained in the interview guide. ${ }^{29}$

\section{Analysis}

The first three study aims involve analyses of the primary binary outcome (the presence or absence of formal/informal ACP one year after the intervention) with different groups of patients and comparisons. We will fit two separate generalized linear mixed models, one for African Americans $(n=400)$ and one for whites $(n=400)$. Each model will have an indicator variable for the structured ACP approach versus the patient-driven, self-management approach, and a cliniclevel random effect to capture the intraclass correlation. We will use a single generalized linear mixed model to compare 
Table 2. Reducing Disparities in the Quality of Palliative Care for Older African Americans through Improved Advance Care Planning Study Measures

\begin{tabular}{|c|c|c|c|c|c|c|c|c|}
\hline \multirow[b]{2}{*}{ Construct } & \multirow[b]{2}{*}{ Measure } & \multicolumn{2}{|c|}{ Participant } & \multicolumn{5}{|c|}{ Schedule of assessment } \\
\hline & & Patient & Surrogate & Baseline & $\begin{array}{l}\text { Three } \\
\text { months }\end{array}$ & $\begin{array}{c}\text { Six } \\
\text { months }\end{array}$ & $\begin{array}{l}\text { One } \\
\text { year }\end{array}$ & $\begin{array}{l}\text { After } \\
\text { death }\end{array}$ \\
\hline Demographics & $\begin{array}{l}\text { Age, gender, race, ethnicity, marital } \\
\text { status, education, employment, } \\
\text { and income adequacy }\end{array}$ & $\mathrm{X}$ & $\mathrm{X}$ & $\mathrm{X}$ & & & & \\
\hline \multirow[t]{5}{*}{$\begin{array}{l}\text { Physical and mental } \\
\text { health, quality } \\
\text { of life }\end{array}$} & $\begin{array}{l}\text { Self-reported health (one item) } \\
\text { Self-reported quality of life } \\
\text { (one item) }\end{array}$ & $X$ & $\mathrm{X}$ & $X$ & $\mathrm{X}$ & $X$ & $\mathrm{X}$ & $\mathrm{X}$ \\
\hline & $\begin{array}{l}\text { In general, would you say your } \\
\text { health [or quality of life] is } \\
\text { excellent, very good, good, fair, } \\
\text { or poor? }\end{array}$ & & & & & & & \\
\hline & Katz ADLs ${ }^{31}$ & $\mathrm{X}$ & & $\mathrm{X}$ & $\mathrm{X}$ & $\mathrm{X}$ & $\mathrm{X}$ & \\
\hline & Promis- $29^{32}$ & $\mathrm{X}$ & & $\mathrm{X}$ & $\mathrm{X}$ & $\mathrm{X}$ & $\mathrm{X}$ & \\
\hline & $\begin{array}{l}\text { Promis Anxiety and Depression } \\
\text { Only }\end{array}$ & & $\mathrm{X}$ & $\mathrm{X}$ & $\mathrm{X}$ & $\mathrm{X}$ & $\mathrm{X}$ & $\mathrm{X}$ \\
\hline $\begin{array}{l}\text { Intensity } \\
\text { of caregiving }\end{array}$ & $\begin{array}{l}\text { Relationship to patient, frequency } \\
\text { of contact, assistance with care, } \\
\text { and medical decision making }\end{array}$ & & $\mathrm{X}$ & $X$ & $\mathrm{X}$ & $\mathrm{X}$ & $\mathrm{X}$ & \\
\hline Health literacy & REALM Short form ${ }^{33}$ & $\mathrm{X}$ & $\mathrm{X}$ & $\mathrm{X}$ & & & & \\
\hline Social support & Lubben Social Network Scale ${ }^{34}$ & $\mathrm{X}$ & $\mathrm{X}$ & $\mathrm{X}$ & & & & \\
\hline \multirow[t]{3}{*}{$\begin{array}{l}\text { Religiosity/ } \\
\text { spirituality }\end{array}$} & $\begin{array}{l}\text { Duke University Religious Index } \\
\text { (DUREL) }^{35}\end{array}$ & $\mathrm{X}$ & $\mathrm{X}$ & $X$ & & & & \\
\hline & Brief R-Cope ${ }^{36}$ & $\mathrm{X}$ & & $\mathrm{X}$ & & & & \\
\hline & $\begin{array}{l}\text { Functional Assessment of Chronic } \\
\text { Illness-Spiritual Well-being } \\
\text { (Facit-Sp) }\end{array}$ & $\mathrm{X}$ & $\mathrm{X}$ & $X$ & & & & \\
\hline Trust $^{\mathrm{a}}$ & $\begin{array}{l}\text { Trust in Provider Scale } \\
\text { Healthcare System Distrust Scale }\end{array}$ & $X$ & $X$ & $X$ & & & & \\
\hline & $\begin{array}{l}\text { Everyday Discrimination Scale } \\
\text { (health care) }^{40}\end{array}$ & & & & & & & \\
\hline $\begin{array}{l}\text { Provider } \\
\quad \text { Communication }\end{array}$ & CAHPS Clinician Survey ${ }^{41}$ & $X$ & $X$ & $X$ & & & & \\
\hline $\begin{array}{l}\text { Desired role in } \\
\text { decision making }\end{array}$ & Control Preferences Scale ${ }^{42}$ & $X$ & & $\mathrm{X}$ & & & & \\
\hline $\begin{array}{l}\text { Prior experience } \\
\text { with medical } \\
\text { decision making }\end{array}$ & $\begin{array}{l}\text { Single item: Have you ever made a } \\
\text { life-threatening medical decision } \\
\text { for yourself or someone else? }\end{array}$ & $X$ & $X$ & $X$ & $X$ & $\mathrm{X}$ & $X$ & \\
\hline \multirow[t]{3}{*}{$\begin{array}{l}\text { Exposure to ACP } \\
\text { information and } \\
\text { knowledge } \\
\text { of } \mathrm{ACP}^{44}\end{array}$} & $\begin{array}{l}\text { How much have you heard about } \\
\text { advance directives? (never heard, } \\
\text { heard a little, fair amount, } \\
\text { or a lot) }\end{array}$ & $\mathrm{X}$ & & $X$ & & & & \\
\hline & $\begin{array}{l}\text { Have you ever received advance } \\
\text { directive forms to complete? }\end{array}$ & & & & & & & \\
\hline & $\begin{array}{l}\text { How knowledgeable would you say } \\
\text { you are about advance directives? } \\
\text { (not at all, a little, somewhat, } \\
\text { fairly, or very knowledgeable) }\end{array}$ & & & & & & & \\
\hline Barriers to $\mathrm{ACP}$ & $\begin{array}{l}\text { Checkbox of common barriers } \\
\text { to } \mathrm{ACP}^{45}\end{array}$ & $X$ & & $X$ & & & & \\
\hline \multicolumn{9}{|c|}{ to ACr } \\
\hline \multirow[t]{2}{*}{$\begin{array}{l}\text { Primary: formal } \\
\text { and informal } \\
\text { ACP }\end{array}$} & $\begin{array}{l}\text { Self-report: items assessing whether } \\
\text { patient has talked to surrogate } \\
\text { about preferences or asked } \\
\text { someone to serve as decision } \\
\text { maker. }\end{array}$ & $\mathrm{X}$ & & $\mathrm{X}$ & $\mathrm{X}$ & $\mathrm{X}$ & $X$ & \\
\hline & $\begin{array}{l}\text { Chart Review (advance directives, } \\
\text { clinician discussions about } \\
\text { preferences) }\end{array}$ & $\mathrm{X}$ & & $X$ & & & $\mathrm{X}$ & $\mathrm{X}$ \\
\hline ACP engagement & $\begin{array}{l}\text { Four-item ACP Engagement } \\
\text { Survey }{ }^{46}\end{array}$ & $\mathrm{X}$ & & $\mathrm{X}$ & $\mathrm{X}$ & $\mathrm{X}$ & $X$ & \\
\hline $\begin{array}{l}\text { Beliefs about } \\
\text { dying and ACP }\end{array}$ & $\begin{array}{l}\text { Beliefs about Dying, Truth Telling, } \\
\text { and Advance Care Planning } \\
\text { Scale }\end{array}$ & $\mathrm{X}$ & & $X$ & $X$ & $X$ & $X$ & \\
\hline
\end{tabular}


TABle 2. (CONTINUED)

\begin{tabular}{|c|c|c|c|c|c|c|c|c|}
\hline \multirow[b]{2}{*}{ Construct } & \multirow[b]{2}{*}{ Measure } & \multicolumn{2}{|c|}{ Participant } & \multicolumn{5}{|c|}{ Schedule of assessment } \\
\hline & & Patient & Surrogate & Baseline & $\begin{array}{l}\text { Three } \\
\text { months }\end{array}$ & $\begin{array}{c}\text { Six } \\
\text { months }\end{array}$ & $\begin{array}{l}\text { One } \\
\text { year }\end{array}$ & $\begin{array}{l}\text { After } \\
\text { death }\end{array}$ \\
\hline $\begin{array}{l}\text { Patient-surrogate } \\
\text { congruence } \\
\text { regarding } \\
\text { patient's care } \\
\text { preferences }^{\mathrm{a}}\end{array}$ & $\begin{array}{l}\text { General Values Scale } \\
\text { Modified Medical Directive }^{48}\end{array}$ & $X$ & $\mathrm{X}$ & $X$ & $X$ & $X$ & $X$ & \\
\hline $\begin{array}{l}\text { Goal-concordant } \\
\text { care }^{49, \mathrm{a}}\end{array}$ & $\begin{array}{l}\text { If you had to make a choice today in } \\
\text { your current health, would you } \\
\text { prefer (1) treatment focused on } \\
\text { extending life as much as } \\
\text { possible, even if it means having } \\
\text { more pain or discomfort or (2) } \\
\text { plan of care that focuses on } \\
\text { relieving pain and discomfort as } \\
\text { much as possible even if it means } \\
\text { not living as long? } \\
\text { Would you say your current } \\
\text { medical care is focused on (1) } \\
\text { extending life as much as } \\
\text { possible even if it means having } \\
\text { more pain and discomfort or (2) } \\
\text { relieving pain and discomfort as } \\
\text { much as possible even if it means } \\
\text { not living as long? }\end{array}$ & $\mathrm{X}$ & $X$ & $X$ & $X$ & $X$ & $X$ & $X$ \\
\hline $\begin{array}{l}\text { Belief that others } \\
\text { know wishes }\end{array}$ & $\begin{array}{l}\text { How confident are you that if you } \\
\text { were unconscious or in a coma } \\
\text { that your doctor [family and } \\
\text { friends] would know what you } \\
\text { want done for you? }\end{array}$ & $\mathrm{X}$ & & $X$ & $X$ & $X$ & $\mathrm{X}$ & \\
\hline $\begin{array}{l}\text { Surrogate comfort } \\
\text { with decision } \\
\text { making, }\end{array}$ & Agent Comfort Questionnaire ${ }^{48}$ & & $X$ & $X$ & $\mathrm{X}$ & $X$ & $\mathrm{X}$ & $\mathrm{X}$ \\
\hline $\begin{array}{l}\text { Intervention } \\
\text { evaluation }\end{array}$ & $\begin{array}{l}\text { Investigator developed measure of } \\
\text { communication with } \\
\text { interventionist, effectiveness of } \\
\text { intervention, and intervention } \\
\text { materials }\end{array}$ & $X$ & & & $X$ & & & \\
\hline $\begin{array}{l}\text { Quality of end-of- } \\
\text { life care }\end{array}$ & $\begin{array}{l}\text { NHATs Last Month of Life }{ }^{50} \\
\text { After death Bereaved Family } \\
\text { Interview }\end{array}$ & & $\mathrm{X}$ & & & & & $\mathrm{X}$ \\
\hline
\end{tabular}

${ }^{\text {a }}$ Surrogate answers based on his/her beliefs about patient experiences or preferences.

the effectiveness of the two ACP approaches in reducing racial disparities in ACP. This model will have an indicator variable for intervention group, a clinic-level random effect, and an indicator variable for race and the interaction between intervention group and race.

To examine the impact of racial concordance of the ACP facilitator on ACP, we will use generalized linear mixed models within four different subgroups (African Americans and whites within each of the two intervention groups). Each model will include an indicator variable for racially concordant versus discordant ACP facilitator and a clinic-level random effect. Finally, we will assess patient and caregiver outcomes for the subsample of patients who die during the study period with models that include an indicator variable for intervention group, a clinic-level random effect, an indicator variable for race, and the interaction between intervention group and race.

\section{Sample size and power considerations}

Our primary hypothesis is that, within each racial subgroup, a structured ACP approach will result in higher rates of ACP by one year than the patient-guided, self-management approach. Based on prior research, we assumed a rate of formal/informal ACP in the patient-driven, self-management ACP group ranging from a low of $20 \%$ to a high of $40 \%$. Calculations assumed a type-I error of 5\% and were completed using tests for two proportions in a cluster randomized design to account for intracluster correlations ranging from 0.01 to $0.02 .^{30} \mathrm{As}$ described above, ACP will be ascertained from both selfreport and by chart review over the one-year study period; therefore, we anticipate complete data on this outcome for all study participants. For each racial subgroup of 400 patients, we will have $80 \%$ power to detect minimum differences of $14.5 \%-18.4 \%$ in rates of ACP between the two ACP 
approaches. To examine racial disparities between the two ACP approaches, the effective sample size of this comparison is one-quarter the overall sample size. Assuming racial disparities in ACP rates ranging from $10 \%$ to $30 \%$, we will have $80 \%$ power to detect differences in reduced disparities of 9\%-18\% between the two ACP approaches. Finally, for our continuous caregiver outcomes among decedents (e.g., satisfaction with end-of-life care), calculations were completed using tests for two means in a cluster randomized design; we will have $80 \%$ power to detect differences in effect sizes of 0.4-0.50 in racial disparities between the two ACP approaches.

\section{Fidelity Monitoring}

Our interventionists are lay ACP facilitators with no prior formal training. We developed a multipronged approach to monitor intervention fidelity. First, all interventionists completed standardized training. The lay ACP facilitators for Respecting Choices First Steps completed the corresponding ACP Facilitator Certification course. ACP facilitators working with participants from clinics in the other study arm completed training in using the scripted protocol that we developed for reviewing the Five Wishes materials and answering questions. Interventionists in both arms completed training in motivational interviewing, general principles of ACP, and completion of advance directive forms. Total inperson instruction ranged from 12 to 14 hours.

Second, during the four to six weeks after the in-person training, ACP facilitators practiced delivering the intervention with research staff at each local site, who had also completed training. After a series of practice sessions, the ACP facilitators were required to deliver the full intervention to a patient or stakeholder and demonstrate successful mastery $(80 \%)$ of competencies relevant to their training.

Third, interventionists in each arm participate in separate monthly group calls where they share experiences delivering the intervention and receive feedback from research staff and instructors. Finally, ACP facilitators will record a random sample of their interventions throughout the course of the study. These will be reviewed using prespecified criteria to assess adherence to the study protocol and provide individual feedback.

\section{Patient and Key Stakeholder Engagement}

Our stakeholder engagement strategy ensures we solicit diverse perspectives from each of the study sites to inform and guide the study. EQUAL ACP has six local Stakeholder Advisory Councils (SACs) - four of them linked to the participating academic institutions and one for each of the South Carolina federally qualified health centers - and a central Stakeholder Advisory Board (SAB). Each SAC and the central SAB consist of 8-10 members, including African American and white patients and caregivers, clinicians, clergy, community educators, and health system leaders. The SAB includes representatives from national- and community-based organizations that aim to promote equitable care. All stakeholders participated in a one-hour webinar, Research 101, which provides information about the research process and stakeholder engagement.

To facilitate bidirectional communication and consistency across sites, we created standardized processes for engagement. SAC and SAB meetings (90 minutes) occur quarterly (in person and Webex) at each site and are facilitated by a member of the local research team. The research team develops a common agenda for all meetings and facilitators participate in a premeeting session to discuss the agenda. SAC facilitators record recommendations and input on a standardized form. These data are compiled and presented at the SAB meetings. Recommendations from the SAB are shared with full research team and local SAC members. During and between meetings, stakeholders offer input on study design, participant recruitment, interventionist training, measures, study materials, and dissemination of information about the study.

\section{Potential Barriers to Study Success}

The primary challenges to study success are participant attrition, loss of interventionists, maintenance of intervention fidelity (see Fidelity Monitoring section), and coordination of data collection. To reduce attrition, we will review the electronic medical record regularly to update contact information for participants, mail reminders to participants two weeks before data collection, and provide participants with a $\$ 50$ incentive for each completed assessment. To address possible loss of interventionists, we will maintain at least four interventionists per site (two from each racial group) with training in Respecting Choices First Steps, and six interventionists (three from each racial group) who are trained in the patient-driven, self-management ACP protocol that can be delivered by telephone to patients across sites. To ensure the timely training of new interventionists, we have access to instructors for both study arms. To address potential issues with data management, we have developed standardized processes for data collection, organization, and storage, training of research personnel, and troubleshooting information technology issues locally and centrally by the EQUAL ACP Operations Team at Duke.

\section{Dissemination}

For the academic community, we will present our finding at scientific meetings and in peer-reviewed journals. Our stakeholders will assist us with implementing dissemination strategies that target their realms of influence, including health care organization and community groups. We will work with stakeholders to develop information that can be adapted for diverse audiences, with particular emphasis on cultural tailoring of information for African Americans and those with low literacy. Our efforts will be augmented by partnerships with local community engagement groups (e.g., CTSA), with established networks for widespread dissemination of information across diverse platforms. Based on our findings, our stakeholders will assist us with identifying next steps, which may include refinement of interventions, replication of study in other settings, or widespread dissemination in local health systems.

\section{Discussion}

ACP may significantly reduce racial disparities in the quality of end-of-life care. To our knowledge, EQUAL ACP is the first large, multisite study to compare the effectiveness of two evidence-based and/or widely used interventions to facilitate ACP and improve end-of-life care for older African 
Americans. The EQUAL ACP protocol was developed based on an extensive review of the literature and stakeholder input to identify historical, interpersonal, community, and system factors that may influence ACP. Although EQUAL ACP cannot address all of these factors, we identified several opportunities to improve ACP for African Americans within the context of existing interventions, including (1) a greater emphasis on the importance of ACP conversations with surrogates and providers even if patients do not complete legal documents; (2) flexibility in approaches to ACP based on individual preferences; (3) and the use of peer-to-peer interactions with lay ACP interventionists to build trust, increase ACP knowledge, and facilitate ACP. In addition, our protocol includes a qualitative component to describe the ACP experience of participants, identify gaps in existing interventions, and elucidate additional strategies or elements that assist with ACP. This information may be used in future studies to ensure that interventions address the range of factors important to African Americans. The findings of EQUAL ACP will inform efforts to develop and implement evidencebased palliative care interventions that promote equitable palliative care outcomes for older, seriously ill African Americans and their caregivers.

\section{Acknowledgment}

Funding: This study is funded by the Patient-Centered Outcomes Research Institute (Contract R-1609-36381).

\section{Author Disclosure Statement}

No competing financial interests exist.

\section{References}

1. Administration for Community Living and Administration on Aging: 2017 Profile of Older Americans. US Department of Health and Human Services, Washington, DC, 2017.

2. Ritchie CS, Zulman DM: Research priorities in geriatric palliative care: Multimorbidity. J Palliat Med 2013;16: 843-847.

3. Morrison RS: Research priorities in geriatric palliative care: An introduction to a new series. J Palliat Med 2013; 16:726-729.

4. Kaiser Family Foundation: Profile of Medicare beneficiaries by race and ethnicity: A Chart Pack 2016. http:// kff.org/report-section/profile-of-medicare-beneficiaries-byrace-and-ethnicity-chartpack 2016 (Last accessed December 18, 2016).

5. US Census Bureau: Statistical Abstract of the United States: 2012, US Census Bureau, Washington, DC, 2012.

6. Williams DR, Priest N, Anderson NB: Understanding associations among race, socioeconomic status, and health: Patterns and prospects. Health Psychol 2016;35:407.

7. Sanders JJ, Robinson MT, Block SD: Factors impacting advance care planning among African Americans: Results of a systematic integrated review. J Palliat Med 2016;19:202-227.

8. Welch LC, Teno JM, Mor V: End-of-life care in black and white: Race matters for medical care of dying patients and their families. J Am Geriatr Soc 2005;53:1145-1153.

9. Dying in America: improving quality and honoring individual preferences near the end of life. Milit Med 2015; 180:365-367.
10. Johnson KS: Racial and ethnic disparities in palliative care. J Palliat Med 2013;16:1329-1334.

11. Song M-K, Ward SE, Fine JP, et al.: Advance care planning and end-of-life decision making in dialysis: A randomized controlled trial targeting patients and their surrogates. Am J Kidney Dis 2015;66:813-822.

12. LoPresti MA, Dement F, Gold HT: End-of-life care for people with cancer from ethnic minority groups: A systematic review. Am J Hosp Palliat Med 2016;33:291-305.

13. Imai K, King G, Nall C: The essential role of pair matching in cluster-randomized experiments, with application to the Mexican universal health insurance evaluation. Stat Sci 2009;24:29-53.

14. Hedeker D, Gibbons RD: Longitudinal Data Analysis. Vol. 451: John Wiley \& Sons, Hoboken, NJ, 2006.

15. Periyakoil VS, Neri E, Kraemer H: No easy talk: A mixed methods study of doctor reported barriers to conducting effective end-of-life conversations with diverse patients. PLoS One 2015;10:e122321.

16. Scott AJ, Wilson RF: Social determinants of health among African Americans in a rural community in the Deep South: An ecological exploration. Rural Remote Health 2011;11: 1634.

17. Kelley AS, Covinsky KE, Gorges RJ, et al.: Identifying older adults with serious illness: A critical step toward improving the value of health care. Health Serv Res 2017; 52:113-131.

18. Moss AH, Lunney JR, Culp S, et al.: Prognostic significance of the "surprise" question in cancer patients. J Palliat Med 2010;13:837-840.

19. Lakin JR, Robinson MG, Bernacki RE, et al.: Estimating 1year mortality for high-risk primary care patients using the "surprise" question. JAMA Intern Med 2016;176:18631865.

20. Callahan CM, Unverzagt FW, Hui SL, et al.: Six-item screener to identify cognitive impairment among potential subjects for clinical research. Med Care 2002;40:771-781.

21. Pecanac KE, Repenshek MF, Tennenbaum D, Hammes BJ: Respecting Choices ${ }^{\circledR}$ and advance directives in a diverse community. J Palliat Med 2014;17:282-287.

22. Huang C-HS, Crowther M, Allen RS, et al.: A pilot feasibility intervention to increase advance care planning among African Americans in the deep south. J Palliat Med 2016;19:164-173.

23. Detering KM, Hancock AD, Reade MC, Silvester W: The impact of advance care planning on end of life care in elderly patients: Randomised controlled trial. BMJ 2010; 340:c1345.

24. Hammes BJ, Rooney BL: Death and end-of-life planning in one midwestern community. Arch Intern Med 1998;158: 383-390.

25. MacKenzie MA, Smith-Howell E, Bomba PA, Meghani $\mathrm{SH}$ : Respecting choices and related models of advance care planning: A systematic review of published evidence. Am J Hosp Palliat Med 2018;35:897-907.

26. Aging with Dignity: Five Wishes (2016). https://fivewishes .org/Home (last accessed September 6, 2016).

27. Harris PA, Taylor R, Thielke R, et al.: Research electronic data capture (REDCap) - A metadata-driven methodology and workflow process for providing translational research informatics support. J Biomed Inform 2009;42:377-381.

28. Friese S: Qualitative Data Analysis with ATLAS. ti. Sage, Thousand Oaks, CA, 2014.

29. Hsieh H-F, Shannon SE: Three approaches to qualitative content analysis. Qual Health Res 2005;15:1277-1288. 
30. Hintze J: PASS 13 Power Analysis and Sample Size Software. LLC Kaysville, UT: NCSS, 2014.

31. Katz S, Downs TD, Cash HR, Grotz RC: Progress in development of the index of ADL. Gerontologist 1970;10(1 Part_1):20-30.

32. Ader DN: Developing the patient-reported outcomes measurement information system (PROMIS). Medical Care 2007;45:S1-S2.

33. Arozullah AM, Yarnold PR, Bennett CL, et al.: Development and validation of a short-form, rapid estimate of adult literacy in medicine. Med Care 2007;45:1026-1033.

34. Lubben J, Blozik E, Gillmann G, et al.: Performance of an abbreviated version of the Lubben Social Network Scale among three European community-dwelling older adult populations. Gerontologist 2006;46:503-513.

35. Koenig HG, Büssing A: The Duke University Religion Index (DUREL): A five-item measure for use in epidemological studies. Religions 2010;1:78-85.

36. Pargament K, Feuille M, Burdzy D: The Brief RCOPE: Current psychometric status of a short measure of religious coping. Religions 2011;2:51-76.

37. Munoz AR, Salsman JM, Stein KD, Cella D: Reference values of the functional assessment of chronic illness therapy-spiritual well-being: A report from the American Cancer Society's studies of cancer survivors. Cancer 2015; 121:1838-1844.

38. Dugan E, Trachtenberg F, Hall MA: Development of abbreviated measures to assess patient trust in a physician, a health insurer, and the medical profession. BMC Health Serv Res 2005;5:64.

39. Shea JA, Micco E, Dean LT, et al.: Development of a revised health care system distrust scale. J Gen Intern Med 2008;23:727-732.

40. Peek ME, Nunez-Smith M, Drum M, Lewis TT: Adapting the everyday discrimination scale to medical settings: Reliability and validity testing in a sample of African American patients. Ethn Dis 2011;21:502.

41. Dyer N, Sorra JS, Smith SA, et al.: Psychometric properties of the Consumer Assessment of Health care Providers and Systems $\left(\right.$ CAHPS $\left.^{\circledR}\right)$ clinician and group adult visit survey. Med Care 2012;50(Suppl):S28.

42. Degner LF, Sloan JA, Venkatesh P: The control preferences scale. Can J Nurs Res Arch 1997;29.
43. Fried TR, Redding CA, Robbins ML, et al.: Stages of change for the component behaviors of advance care planning. J Am Geriatr Soc 2010;58:2329-2336.

44. Johnson KS, Kuchibhatla M, Tulsky JA: What explains racial differences in the use of advance directives and attitudes toward hospice care? J Am Geriatr Soc 2008;56:1953-1958.

45. Schickedanz AD, Schillinger D, Landefeld CS, et al.: A clinical framework for improving the advance care planning process: Start with patients' self-identified barriers. J Am Geriatr Soc 2009;57:31-39.

46. Sudore RL, Heyland DK, Barnes DE, et al.: Measuring advance care planning: Optimizing the advance care planning engagement survey. J Pain Symptom Manage 2017; 53:669-681.e668.

47. Winter L: Patient values and preferences for end-of-life treatments: Are values better predictors than a living will? J Palliat Med 2013;16:362-368.

48. Schwartz CE, Wheeler HB, Hammes B, et al.: Early intervention in planning end-of-life care with ambulatory geriatric patients: Results of a pilot trial. Arch Intern Med 2002;162:1611-1618.

49. Connors AF, Dawson NV, Desbiens NA, et al.: A controlled trial to improve care for seriously iII hospitalized patients: The study to understand prognoses and preferences for outcomes and risks of treatments (SUPPORT). JAMA 1995;274:1591-1598.

50. Teno JM, Freedman VA, Kasper JD, et al.: Is care for the dying improving in the United States? J Palliat Med 2015; 18:662-666.

51. Teno JM, Clarridge B, Casey V, et al.: Validation of toolkit after-death bereaved family member interview. J Pain Symptom Manage 2001;22:752-758.

Address correspondence to: Deborah B. Ejem, PhD Division of Preventive Medicine School of Medicine University of Alabama at Birmingham 1720 2nd Avenue South Birmingham, AL 35294

E-mail: tejem@uab.edu 